\title{
Tchir, Trevor: Hannah Arendt's Theory of Political Action. Daimonic Disclosure of the 'Who', 258 S., Palgrave Macmillan, Basingstoke/New York 2017.
}

\author{
Karl-Heinz Breier \\ Online publiziert: 9. Juni 2020 \\ (C) Der/die Autor(en) 2020
}

Wer das Buch von Trevor Tchir, einem kanadischen Politikwissenschaftler der Algoma University, gelesen hat, kommt nicht umhin, Hannah Arendts Vita activa erneut in die Hand zu nehmen und ihr fünftes Kapitel über „Das Handeln“ aufzuschlagen. Dort erörtert die politische Denkerin unser originär menschliches Vermögen zu sprechen und zu handeln, und im Zentrum steht dabei die Enthüllung unserer Person. Im Unterschied zu anderen Tätigkeiten, die in der Bearbeitung der äußeren Natur und im Herstellen von Dingen der Gegenstandswelt als materialgebundene Leistungen in Erscheinung treten, geben das Sprechen und das Handeln Aufschluss über das Wer der jeweiligen Person. Die Einzigartigkeit der Person zeigt sich im Sprechen und im Handeln, und es ist diese Enthüllung der Person, die originär politischen Charakter hat. Denn um das unverwechselbare Wer eines Menschen, seinen daimon, zu sehen, bedarf es eines weltlichen Erscheinungsraumes. Es bedarf der Bühne der Welt, die - politisch gesprochen - sich als Raum handlungsfreundlicher Institutionen präsentiert.

In dieser Arendt'schen Perspektive thematisiert Tchir den Zusammenhang von Person und Institution, der zwar einerseits seinem gesamten Gedankengang zugrunde liegt, der aber andererseits stets anthropologisch konnotiert ist. So zeichnet der Autor etwa im mittleren Kapitel seines Buches umrisshaft Martin Heideggers Daseinsanalyse nach. Dies geschieht sicherlich in der Absicht, Hannah Arendts denkerische Wurzeln freizulegen. Aber hier - im vierten Kapitel - spürt Tchir zugleich ihrem antiplatonischen Denken nach, wenn er Wahrheit (aletheia) im Heidegger'schen Sinne als Unverborgenheit auffasst. Wahrheit wird demzufolge nicht auf objektivierbare Richtigkeit enggeführt, sondern ganz in phänomenologischem Sinne als sich entbergendes Geschehen interpretiert, als die Person enthüllendes Geschehen.

Auf der anderen Seite jedoch zeigt Tchir auch auf, wo Arendt Heidegger nicht mehr folgt, nämlich in der Interpretation von Öffentlichkeit, in deren Erörterung Heidegger im durchschnittlichen „Man“ (the they) geradezu das die Person Verschließende und die Authentizität der Person Negierende hervorhebt. „Arendt's Answer to Heidegger's Authentic Dasein“ (S. 115-119) führt nach Tchir vielmehr zu Aris-

K.-H. Breier (ه)

Universität Vechta, Vechta, Deutschland

E-Mail: karl-heinz.breier@uni-vechta.de 
toteles zurück, denn ,human beings are only eudaimon at the end of a complete life."

Mit dem eigenen daimon, der sich allerdings nur der Mitwelt zeigt, in Übereinstimmung zu leben, bedeutet nach griechischem Verständnis eudaimonia, eben gelungene Existenz. So ist es spannend und auch sehr variantenreich entfaltet, wie Tchir anhand des Begriffs daimon Arendts Verknüpfung von Selbst und Welt ausleuchtet, wie sie etwa Heidegger ins Politische wendet, indem sie die Heidegger'sche Weltvergessenheit kategorial überwindet, oder wie sie anhand der erweiterten Urteilskraft von Immanuel Kant - „Politicizing Kant's Aesthetic Judgment“ (S. 171-203) die „Dignity of Doxa“ rehabilitiert. Denn urteilen heißt, das Besondere aufzufassen.

Indem wir Menschen als sprechende und handelnde Wesen sowohl uns selbst in unserer personalen Einmaligkeit offenbaren als auch Aufschluss geben über die Welt, in der wir agieren, werden wir zu Urhebern unserer eigenen Existenz. Jedoch darf diese Autorenschaft unserer Lebensgeschichte nicht verwechselt werden mit der Art von Urheberschaft, wie sie der Verfasser einer Novelle oder eines Theaterstückes für sich beanspruchen kann. Denn im Unterschied zu der vom Autor ersonnenen und verfassten Geschichte sind die Geschichten handelnder Wesen in ihrem Fortgang unabsehbar und damit offen. So ergibt es Sinn, dass Tchir sein Buch mit „Arendt's Critique of Teleological Philosophies of History“ (S. 205-233) schließt, indem er „Forgotten Fragments“ ans Licht befördert. Dabei stützt er sich vor allem auf bisher noch nicht veröffentlichte Vorlesungsaufzeichnungen von Arendt, die als „Hannah Arendt Papers“ in der Library of Congress im Original und als Kopie in der Universität Oldenburg aufbewahrt werden.

Als phänomenologisch argumentierende Denkerin versteht Arendt das Politische von den Phänomenen her, und in dieser, sie auszeichnenden, Denkungs- und Verstehensart tritt das Politische in seiner Besonderheit zutage. In dieser Hinsicht so Tchir - unternimmt es Arendt, die menschlichen Angelegenheiten, die schon immer interpretiert und sinnhaft ausgedeutet sind, in freiheitlichen Sinn- und Bedeutungszusammenhängen auszulegen. Nicht das sozialwissenschaftlich und typologisch erfassbare Was von Menschen wird erörtert, sondern wer jemand in concreto ist. Denn als jeweils handelndes Wer vollziehen wir unsere zweite Geburt. So verändern wir mit unserem Handeln, wenn wir die Welt als Ort und Gestaltungsraum unserer Aktivitäten wahrnehmen, nicht nur all die weltlichen Bezüge, in denen wir leben. Indem wir uns vielmehr in das weltliche Bezugsgewebe einweben und einbinden, kommen wir selbst auch erst zur Welt. Jeder Einzelne wird zur Person, zur Person mit unverwechselbarer Biografie. Das heißt: Neben der biologischen Geburt erfahren wir eine biografische Geburt. Neben der Geburt als Lebewesen erfahren wir unsere Geburt als Weltwesen, und zwar wodurch? Durch unser Tun, durch die konkrete Art und Weise unseres Tätigseins, durch unsere Vita activa. Zu leben heißt Tätigsein, und originär menschlich zu leben, heißt im Lateinischen inter homines esse, unter Menschen anwesend zu sein. Damit sind wir Menschen nicht nur in der Welt, so wie alle in der Welt herumstehenden Dinge etwa in der Welt sind, sondern als originäre Weltwesen, die im Handeln zur Anwesenheit drängen, sind wir von der Welt, wie Arendt sagt. Und dieser Sorge um die Welt - so können wir mit Trevor Tchir weiterdenken - gilt Hannah Arendts denkerische Aufmerksamkeit. 
Funding Open Access funding provided by Projekt DEAL.

Open Access Dieser Artikel wird unter der Creative Commons Namensnennung 4.0 International Lizenz veröffentlicht, welche die Nutzung, Vervielfältigung, Bearbeitung, Verbreitung und Wiedergabe in jeglichem Medium und Format erlaubt, sofern Sie den/die ursprünglichen Autor(en) und die Quelle ordnungsgemäß nennen, einen Link zur Creative Commons Lizenz beifügen und angeben, ob Änderungen vorgenommen wurden.

Die in diesem Artikel enthaltenen Bilder und sonstiges Drittmaterial unterliegen ebenfalls der genannten Creative Commons Lizenz, sofern sich aus der Abbildungslegende nichts anderes ergibt. Sofern das betreffende Material nicht unter der genannten Creative Commons Lizenz steht und die betreffende Handlung nicht nach gesetzlichen Vorschriften erlaubt ist, ist für die oben aufgeführten Weiterverwendungen des Materials die Einwilligung des jeweiligen Rechteinhabers einzuholen.

Weitere Details zur Lizenz entnehmen Sie bitte der Lizenzinformation auf http://creativecommons.org/ licenses/by/4.0/deed.de.

\title{
Löschenkohl, Birte: Freiheit und Wiederholung. Politisches Handeln ohne Zukunft, 236 S., Fink, München/Paderborn 2018.
}

\author{
Matthias Gsänger \\ Online publiziert: 20. April 2020 \\ (C) Der/die Autor(en) 2020
}

Die Schwierigkeiten, mittels zielorientierten politischen Handelns bestimmte gesellschaftliche Zustände zu erreichen, waren schon Gegenstand der Steuerungsdebatte Anfang der 1990er Jahre. Dass die Probleme nicht kleiner werden, wenn es statt um policies, wie dem Ausbau von Kita-Plätzen, innerhalb einer bestehenden politischen Ordnung um grundlegende Veränderungen dieser Ordnung selbst geht, ist ein Ausgangspunkt von Birte Löschenkohls Buch. Ein anderer ist die Unplausibilität sowohl der Annahme souveränen Handelns als auch der Vorstellung einer quasi automatischen Entwicklung der Menschheit zum Besseren. Die Autorin sieht die Notwendigkeit, den Charakter von Praxisvollzügen umzudenken, und unterbreitet in ihrer Argumentation den Vorschlag, ,,das Potential der Wiederholung für die Entwicklung einer solchen konzeptuellen Alternative zu erkunden“ (S. 11). Sie unterscheidet zwischen

\footnotetext{
M. Gsänger $(\bowtie)$

Universität Würzburg, Würzburg, Deutschland

E-Mail: matthias.gsaenger@uni-wuerzburg.de
} 